\title{
Miami Classification for Probe-Based Confocal Laser Endomicroscopy
}

\section{Citation}

Wallace, M., G. Lauwers, Y. Chen, E. Dekker, P. Fockens, P. Sharma, and A. Meining. 2011. Miami classification for probe-based confocal laser endomicroscopy. Endoscopy 43(10): 882-891.

\section{Published Version}

doi:10.1055/s-0030-1256632

\section{Permanent link}

http://nrs.harvard.edu/urn-3:HUL.InstRepos:12601541

\section{Terms of Use}

This article was downloaded from Harvard University's DASH repository, and is made available under the terms and conditions applicable to Other Posted Material, as set forth at http:// nrs.harvard.edu/urn-3:HUL.InstRepos:dash.current.terms-of-use\#LAA

\section{Share Your Story}

The Harvard community has made this article openly available.

Please share how this access benefits you. Submit a story.

Accessibility 


\section{Miami classification for probe-based confocal laser endomicroscopy}

Authors

Institutions
M. Wallace' ${ }^{1}$, G. Y. Lauwers ${ }^{2}$, Y. Chen ${ }^{3}$, E. Dekker ${ }^{4}$, P. Fockens ${ }^{4}$, P. Sharma ${ }^{5}$, A. Meining ${ }^{6}$

Institutions are listed at the end of article. submitted 25. August 2010 accepted after revision 15. May 2011

\section{Bibliography}

Dol http://dx.doi.org/

10.1055/s-0030-1256632

Published online: 04.08 .2011

Endoscopy 2011; 43: 882-891

(c) Georg Thieme Verlag KG

Stuttgart · New York

ISSN 0013-726X

\section{Corresponding author}

M. B. Wallace, MD, MPH

Mayo Clinic Jacksonville

4500 San Pablo Road South Jacksonville

FL 32224

USA

Fax: +1-904-953-7260

wallace.michael@mayo.edu
An essential element for any new advanced imaging technology is standardization of indications, terminology, categorization of images, and research priorities. In this review, we propose a state-of-the-art classification system for normal and pathological states in gastrointestinal disease

\section{Introduction}

$\nabla$

Probe-based confocal laser endomicroscopy (pCLE) and its related technology, endoscopebased CLE (eCLE), are rapidly emerging methods of imaging with the potential to fundamentally change the role of biopsy in gastrointestinal endoscopy. Since the advent of endoscopic imaging, biopsy has been used to confirm or exclude pathology. Although highly valuable, biopsy is expensive, and there can be delays between endoscopy and a "confirmed" diagnosis and/or treatment. In addition, although the risks associated with biopsy are small, the number of biopsies performed worldwide is enormous, and therefore risk becomes an important consideration.

CLE methods offer the potential to decrease or possibly eliminate the need for some biopsies and to directly guide treatment of circumscribed lesions, such as colorectal polyps, in real time. CLE can also help to avoid biopsy, or if biopsy is necessary, to direct it much more efficiently in areas of very low diagnostic yield, such as in surveillance of Barrett's esophagus, ulcerative colitis, and indeterminate bile duct strictures. Although it is still premature to determine the exact roles of pCLE in each gastrointestinal condition, it is essential to establish standards for image interpretation at an early stage of development.

We determined that a unique classification system was needed for pCLE due to the significant technical differences compared with eCLE (smaller field of view, fixed depth), as well as the fact that many of the eCLE images published in the literature have used acriflavine (a nuclear stain) and using probe-based confocal laser endomicroscopy (pCLE). The Miami classification system is based on a consensus of pCLE users reached during a meeting held in Miami, Florida, in February 2009.

are not comparable to pCLE images, which are almost exclusively obtained with fluorescein-only contrast.

\section{Methods for development of criteria $\nabla$}

A group of experts was assembled based on their early experience with pCLE imaging in a variety of gastrointestinal conditions. As pCLE technology is new, there was a limited number of individuals with significant expertise in each field prior to February 2009, and a limited number of clinical trials on which to establish standards. Thus the standards were largely based on expert opinion, and consensus development. In general, each group developed criteria based on the following algorithm:

- acquisition and review of unended images with pathological confirmation

- description of features unique to each histological state (e.g. metaplasia, neoplasia)

- pilot testing of accuracy in a small sample of cases blinded to the histology

- where available validation, including inter-observer agreement, in a large sample with multiple users.

The Miami consensus conference involved presentation of standard images and specific features of each condition, together with group discussion and consensus development. Following the conference, a report was drafted by the lead co-author for each section, and the final criteria and manuscript were approved by all co-authors. 


\section{General techniques for pCLE \\ $\nabla$}

\section{Probe technology}

The confocal microscope used in pCLE captures microscopic images of untreated in vivo tissue. The microscope uses focused laser light of a defined wavelength and passes it through a confocal aperture. Images are then reconstructed in two dimensions.

For pCLE (Cellvizio by Mauna Kea Technologies, Paris, France), both the laser scanning unit and light source are outside the body. The laser beam is transported via flexible confocal miniprobes and a distal lens sequentially scans it through a bundle of more than 10000 optical fibers. Confocal miniprobes are flexible, with diameters ranging from $0.9 \mathrm{~mm}$ to $2.5 \mathrm{~mm}$. Therefore, this system may be introduced through the working channels of endoscopes ( $\bullet$ Fig. 1$)$.

\section{Image acquisition, processing, and display}

pCLE image data are collected at 12 frames per second, enabling video quality and direct visualization of blood on a single erythrocyte scale. Depending on the probe used, the field of view ranges from $240 \mu \mathrm{m}$ to $600 \mu \mathrm{m}$ (one-quarter to one-half of a millimeter). The clinical system consists of a miniprobe connected to a laser scanning unit that is connected to a standard personal computer for image data processing and display ( $\bullet$ Fig. 1). Images are reviewed with a specially designed software package (Cellvizio Viewer), allowing image correction and stabilization.

\section{Contrast agents, dose, and safety}

Different dyes, such as fluorescein or cresyl violet, have been used for contrast enhancement [1-3]. Cresyl violet may only be administered topically, whereas fluorescein may also be injected in- travenously. Fluorescein is the current substance of choice because it is relatively inexpensive, non-mutagenic, enables higher imaging depth than topically administered contrast agents, and it has been safely used for decades in ophthalmology [4]. Injection of $1.0-5.0 \mathrm{~mL}$ of a $10 \%$ solution enables visualization of individual cells with strong contrast of the capillary network. This is the current standard dosage in most settings. Hence, dynamic images of blood flow and supply are possible, making it a useful tool for detection of neo-angiogenesis [5].

The exact mechanism by which neoplastic cells appear dark is poorly understood. It is clear that fluorescence is not seen within these cells. Several mechanisms have been proposed including lack of fluorescein uptake, more rapid excretion from the cell [1], or greater leakage into the lamina propria, thus increasing the relative darkness of the epithelial cells [6].

\section{Endoscopic techniques for high-quality image acquisition of mucosal disease}

The keys to obtaining high quality images are timing, positioning, and stability. Gradual changes in image quality occur in a timedependant fashion after intravenous injection of fluorescein. Optimal contrast is obtained within the first $10 \mathrm{~min}$ after injection, but good quality images can be acquired for a further 30-60 min [3]. However, bleeding can impair image quality; therefore, the probe should be placed gently in contact with the tissue in order to avoid trauma, particularly if friability is expected, such as in neoplasms or severely inflamed tissue. As with other imaging methods, it is best to complete pCLE imaging in a region before biopsy or tissue removal is completed.

If possible, the probe should be perpendicular to the mucosa, rather than parallel to it. This can be difficult if the lumen is very
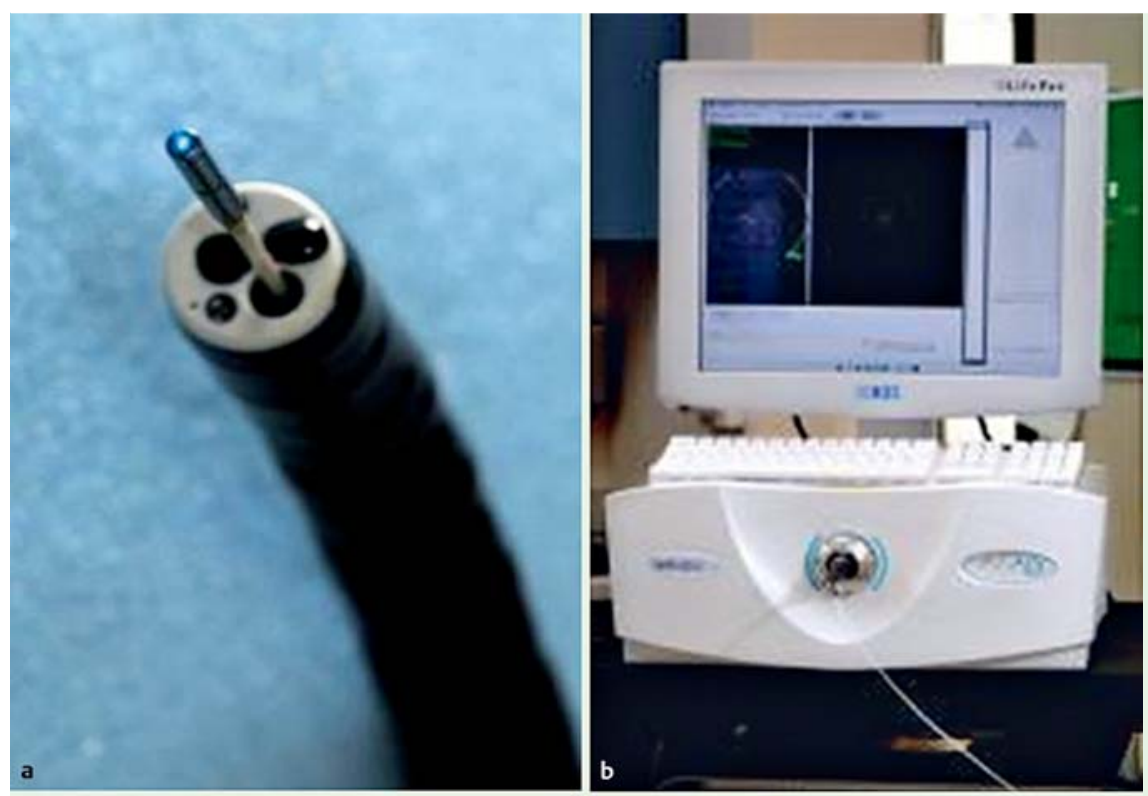

Fig. 1 The probe-based confocal laser endomicroscopy (pCLE) system. a Probe within standard endoscope. $\mathbf{b}$ Laser scanning unit and display. c Fiber probe. $\mathbf{d}$ Fiber probe with blue laser illuminated.
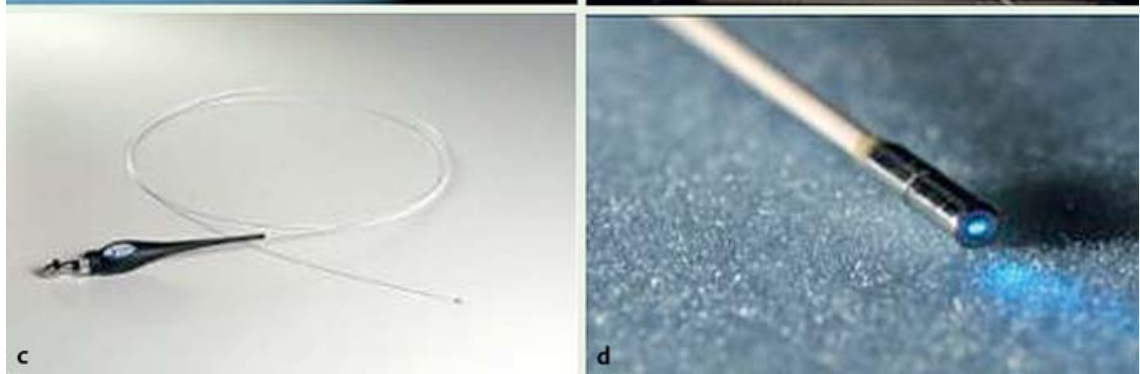
narrow, as in biliary strictures. In these circumstances, a cholangioscopical approach or biliary catheter with side hole or bendable tip might help to maneuver the probe into the wall of the bile duct.

Movements caused by the examiner or the patient (breathing or peristalsis) can significantly impair positioning of the probe and cause artifacts. Using a clear 4-mm cap on the tip of the endoscope and applying gentle suction helps to hold the probe in position. This approach can be very helpful for the imaging of Barrett's esophagus or small colonic polyps.

Other methods to improve the match between pCLE imaging sites and biopsy sites include the use of a dual-channel endoscope (with pCLE probe in one channel and biopsy forceps in the other) or the use of cautery marking with argon plasma coagulation or coagulating ("hot") biopsy forceps. The most useful methods for research studies require highly precise matching of image and biopsy locations.

\section{Applications of pCLE}

The main focus of pCLE has been the detection and classification of neoplasia in Barrett's esophagus and colonic polyps. However, several other conditions have also been studied to a lesser extent and others are just beginning to be studied. Table 1 lists these potential applications in order of the current extent of evidence.

\section{Comparison of eCLE and pCLE}

Both endoscopy- and probe-based methods of CLE have advantages and disadvantages. eCLE offers a wider field of view, the ability to vary the depth of imaging from $0-250 \mu \mathrm{m}$ compared with a fixed imaging plane (approximately $50 \mu \mathrm{m}$ below the surface of GastroFlex), and slightly better lateral resolution (approximately $0.8 \mu \mathrm{m}$ vs. $1 \mu \mathrm{m}$ ). Advantages of pCLE are the versatility of a probe system that can be passed via any endoscope, or even needles, and more rapid image acquisition (12 frames per second vs. 0.81.6 frames/s), which allows imaging of in vivo blood flow. Both systems have inherent limitations compared with wide field endoscopic methods, including the very small field of view, cost of the technology, learning curve, and extra time needed to view the images during endoscopy.

\section{Barrett's esophagus}

$\nabla$

Surveillance guidelines for the management of patients with Barrett's esophagus call for target biopsies from visible lesions, followed by random four-quadrant biopsies every $2 \mathrm{~cm}$ [7]. This practice is time-consuming, costly, and challenging as sampling is not always accurate. Detailed analysis of esophagectomy specimens from patients with Barrett's esophagus has revealed that

Table 1 Potential applications of (probe-based) confocal laser endomicroscopy.

Well-evaluated areas
Barrett's esophagus guide to biopsy
Colon polyp classification
Areas of early exploration
Inflammatory bowel disease dysplasia
Biliary strictures
Duodenal neoplasia
Experimental areas
Solid and cystic tumor imaging
Gastric neoplasia

areas of low-grade dysplasia (LGD), high-grade dysplasia (HGD), and cancer are extremely focal and occupy extremely small areas within the Barrett's segment [8]. To overcome these shortcomings, advanced endoscopic techniques including pCLE have been developed to maximize the positive and negative predictive values of dysplasia detection in patients with Barrett's esophagus. CLE is a highly targeted technology that may be useful for lesion classification. However, other technologies, such as narrow band imaging (NBI), autofluorescence imaging (AFI), and chromoendoscopy are needed as "broad-base surface imaging" techniques to initially detect and localize suspicious areas.

In patients with Barrett's esophagus, CLE captures very high resolution and detailed images including glandular architecture, crypts, columnar cells, goblet cells, and capillaries with red blood cells. In patients with a columnar-lined esophagus, the diagnosis of Barrett's esophagus can be confirmed by identifying and confirming the presence of intestinal metaplasia, which is characterized by the presence of goblet cells. Furthermore, based on preliminary data, CLE also has high accuracy in detecting HGD or cancer.

Classification of CLE images as non-dysplastic or dysplastic has been evaluated in a few pilot studies using varying criteria $[3,4]$. An initial study by Kiesslich et al. evaluated criteria for gastric type, intestinal and neoplastic epithelium, based on cellular and vascular architecture details of CLE images [9]. Images that demonstrate regular-shaped capillaries visible only in deeper mucosa, regular columnar-lined epithelium with round glandular openings, and typical cobblestone appearance, were suggestive of gastric-type foveolar epithelium; whereas, regular capillaries that were present in upper and deeper parts of the mucosal layer along with identification of dark ("non-refractile") mucin in goblet cells in columnar-lined mucosa, were diagnosed as intestinal metaplasia (non-dysplastic Barrett's esophagus). The diagnosis of dysplasia/cancer was based on the identification of irregular capillaries in upper and deeper parts of the mucosal layer (suggestive of neo-angiogenesis) with black cells that had irregular apical and distal borders and shapes on confocal images. Using these criteria, Barretts-associated dysplasia could be predicted with a sensitivity of $93 \%$ and a specificity of $98 \%$. Compared with gastric metaplasia, Barrett's metaplasia could be predicted with a sensitivity of $98 \%$ and a specificity of $94 \%$.

In another recent study, Pohl et al. tested the diagnostic characteristics of pCLE for the detection of invisible Barrett's-associated dysplasia [10]. The investigators initially established pCLE criteria for neoplastic Barrett's esophagus including dark, irregularly thickened epithelial borders, dilated irregular vessels, and in adenocarcinoma, disorganization of villiform structures and crypts and dark columnar cells. These criteria were established from 95 biopsies (15 patients) and then tested prospectively in 201 biopsies ( 23 patients). pCLE videos were also assessed by two endoscopists and the inter-observer agreement was tested. The overall sensitivity, specificity, positive predictive value (PPV) and negative predictive value (NPV) were $80 \%, 94 \%, 44 \%$, and $99 \%$, respectively, with good inter-observer agreement (Kappa $=0.6$ ). A wide group of expert endoscopists evaluated these criteria and validated them as reliable and reproducible.

Using a standardized set of 20 video images of dysplastic [11] and non-dysplastic Barrett's sites [10], Wallace et al. reported preliminary sensitivity of $88 \%$ and specificity of $96 \%$ and good interobserver agreement (agreement rate 86\%, Kappa=0.72) [12]. These pCLE criteria have now been tested and validated in a large, prospective, multi-center, randomized controlled trial (DON'T 


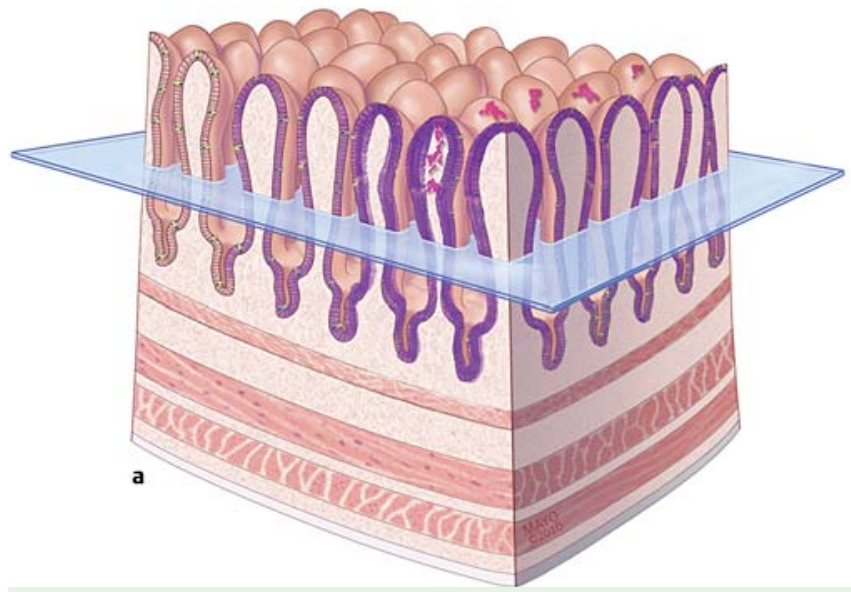

Fig. 2 Esophagus. a Graphic of Barrett's mucosa with villiform non-dysplastic epithelium (left side) progressing to higher grades of dysplasia and cancer (right). The estimated image "slice" of probebased confocal laser endomicroscopy (pCLE) is shown in blue. b pCLE images and histology of esophageal conditions including Barrett's esophagus. In normal squamous epithelium, two pCLE images are shown as well as standard histology with hematoxylin and eosin (H\&E). For Barrett's esophagus, high-grade dysplasia, and adenocarcinoma, a PCLE image is shown on the left and a corresponding $\mathrm{H} \& \mathrm{E}$ image of the same condition is shown on the right.

Normal squamous epithelium
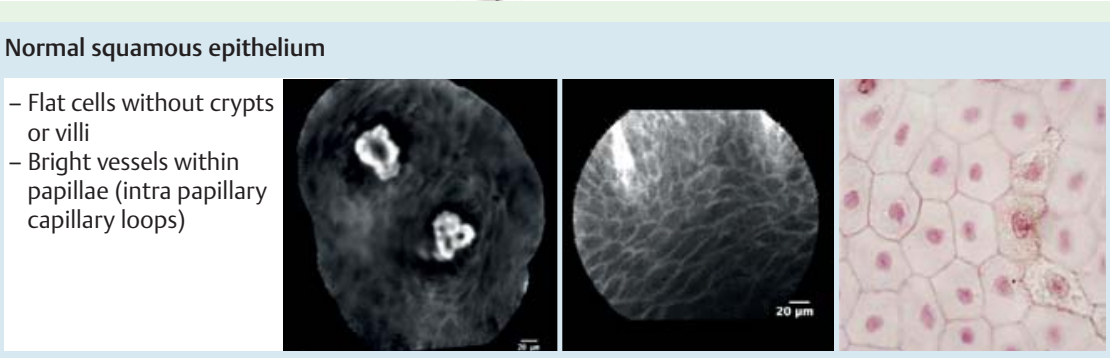

Non dysplastic Barrett's esophagus
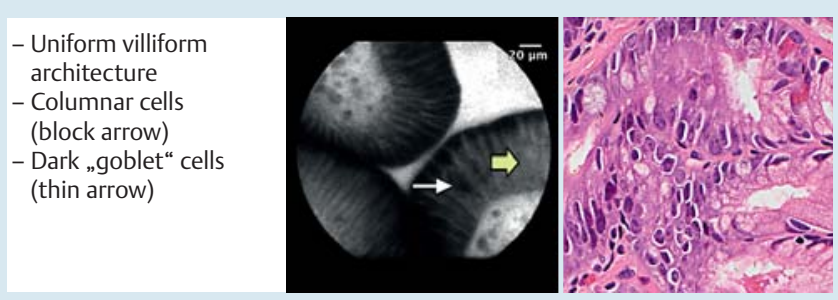

High grade dysplasia

- Villiform structures

- Dark, irregularly thickened epithelial borders (arrow)

- Dilated irregular vessels (block arrow)
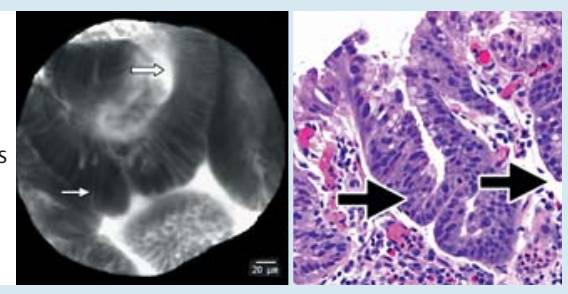

\section{Adenocarcinoma}

$$
\begin{aligned}
& \text { - Disorganized/loss of } \\
& \text { villiform structure and } \\
& \text { crypts } \\
& \text { - Dark columnar cells } \\
& \text { (thin arrow) } \\
& \text { - Dilated irregular vessels } \\
& \text { (block arrow) }
\end{aligned}
$$
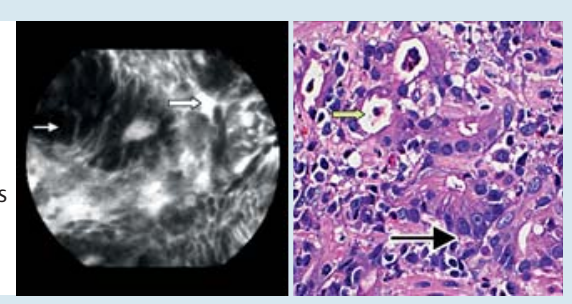

b

BIOPCE trial) [13]. This trial used independent, blinded endoscopists to perform tandem endoscopic procedures to evaluate the sensitivity and specificity of pCLE in addition to white light endoscopy for the detection of HGD and early adenocarcinoma in Barrett's esophagus. Final data presented at Digestive Disease Week 2010 (1-6 May, New Orleans, Louisiana, USA) included 874 sites (120 with high grade intra-epithelial neoplasia [HGIEN] or cancer) in 101 patients ( 31 with HGIEN or cancer). On a per patient basis, the combination of pCLE with high-definition white light endoscopy was significantly more accurate than endoscopy alone (sensitivity/specificity of pCLE + endoscopy 95\%/67\% vs. endoscopy $85 \% / 71 \%$ ). The addition of pCLE to endoscopy and NBI was also more accurate than endoscopy + NBI alone (sensitivity/ specificity of pCLE + endoscopy + NBI 100\%/56\% vs. endoscopy + NBI 97\%/56\%). Using pCLE to guide biopsy in patients with Barrett's esophagus, where only suspicious sites are biopsied would result in a $76 \%$ reduction in biopsies with no HGIEN patients missed, although $24 \%$ of sites would have been missed. If pCLE was 
used to guide biopsy, and four-quadrant random plus targeted biopsies were taken only in those patients with at least one abnormal lesion on pCLE, then this would result in a 39\% reduction in biopsies with no missed sites or patients. In summary, CLE criteria for dysplastic and non-dysplastic Barrett's esophagus have been established and tested in pilot studies. Their validation in a large multicenter study is ongoing and eagerly awaited. It is likely that these criteria will continue to be refined as larger studies are conducted.

- Fig. 2 shows the current pCLE-based classification system with image and histological examples.

\section{Biliary disease \\ $\nabla$}

Accurate diagnosis and staging of pancreatobiliary cancers is important for both prognosis and for guiding therapy; however, it remains challenging to differentiate benign from malignant strictures despite advanced imaging and tissue sampling methods. Up to $15 \%$ of all suspected cholangiocarcinomas originating from the liver hilum reveal benign histology following surgery [11]. Conversely, only $20 \%$ of hilar cancers are resectable at the time of diagnosis, and the rate of R0 resections is unfavorably low [14]. This diagnostic dilemma holds true despite the introduction of tissue sampling methods such as biopsy, brush cytology, or fine-needle aspiration (FNA) cytology through endoscopic retrograde cholangiopancreatography (ERCP) or endoscopic ultrasound (EUS). The best outcomes are obtained when several sampling techniques are combined, but even they remain suboptimal. Rösch et al. reported that combining brush cytology with forceps biopsy yielded a sensitivity of $54 \%$ and a specificity of $100 \%$ [15]. If endoscopic ultrasound-guided fine-needle aspiration (EUS-FNA) was added to these methods, then the sensitivity increased to $71 \%$, but specificity was still $100 \%$. Ponchon et al. reported that combining brush cytology and forceps biopsy yielded a sensitivity of $63 \%$ and a specificity of $97 \%$ [16]. Schoefl et al. showed that the combination of brush cytology and biopsy yielded a sensitivity of $70.4 \%$ and a specificity of $100 \%$ [17]. Hence, there is a critical need to improve the diagnostic accuracy of pancreatobiliary pathology, thereby enabling a more tailored approach for patients and minimizing unnecessary operations.

pCLE that provides in vivo microscopic imaging of tissues in real time can be introduced through the working channel of an ERCP catheter or cholangioscope, in order to access and visualize the pancreatobiliary ductal system during the procedure. Meining et al. reported on a series of 14 patients with biliary strictures that were examined by pCLE via cholangioscopy, and compared the modality to standard endoscopic tissue sampling [18]. pCLE imaging of the biliary stricture was feasible in all cases. The presence of irregular, dilated ("angiogenic") vessels was the laser microscopic hallmark for prediction of neoplasia, with an accuracy of $86 \%$, sensitivity of $83 \%$, and specificity of $88 \%$. The respective numbers for standard histopathology were $79 \%, 50 \%$, and $100 \%$. The mean signal-to-noise-ratio of endomicroscopic images acquired from malignant strictures differed significantly from those of benign origin $(1.8 \pm 0.8$ vs. $2.6 \pm 1.0 ; P=0.005)$. As a preliminary conclusion, pCLE could considerably increase sensitivity for the detection of pancreatobiliary neoplasia, and therefore, represents a promising diagnostic approach. Large multicentric studies are ongoing.

To date, pCLE examination during ERCP procedures has been selectively applied to patients with signs, symptoms, or test results
Table 2 List of endoscopic retrograde cholangiopancreatography (ERCP) devices that have been used successfully and can accommodate the CholangioFlex probe (Cellvizio, Mauna Kea Technologies, Paris). The list is based on clinical experiences of current users of probe-based confocal laser endomicroscopy and does not represent a recommendation from the manufacturers. Differences in size and diameter of the working channel might impair the compatibility in some cases.

\begin{tabular}{|c|c|}
\hline Device name & Manufacturer ${ }^{\mathrm{a}}$ \\
\hline Cotton ${ }^{\circledR}$ Graduated Dilation Catheter T7.0 & Cook Medical \\
\hline $\begin{array}{l}\text { OASIS }{ }^{\circledR} \text { One Action Stent Introduction } \\
\text { System }\end{array}$ & Cook Medical \\
\hline $\begin{array}{l}\text { Memory Dormia basket (Ref MSB_35_2X4 } \\
\text { Memoryll) }\end{array}$ & Cook Medical \\
\hline Howell Biliary Introducer (H-BIN) & Cook Medical \\
\hline Geenen ${ }^{\circledR}$ Graduated Dilation Catheter & Cook Medical \\
\hline Swing Tip ${ }^{T M}$ ERCP cannula & Olympus Medical \\
\hline
\end{tabular}

a Cook Medical, Inc., Bloomington, Indiana, USA; Olympus Medical Systems, Tokyo, Japan

that indicate malignancy or indeterminate pancreatobiliary stricture. The technique for pancreatobiliary pCLE is straightforward. ERCP is performed in accordance with the standard of care, and the stricture is accessed either with an ERCP catheter or a cholangioscope. The pCLE probe is introduced through the lumen of the catheter or the working channel of the cholangioscope, and the lesion of interest is targeted for microscopic imaging using fluoroscopy or endoscopic guidance. The pCLE probe is positioned at the site(s) of suspected pathology immediately following intravenous injection of fluorescein sodium. Real-time in vivo sequences are acquired and stored for review, usually immediately after a site of interest has been imaged.

The choice of the optimal access delivery system for pCLE during ERCP (catheter vs. cholangioscopic access and delivery) is currently being investigated. In part, the decision depends on the endoscopy unit resources and operator preference. Initial experience with pCLE during ERCP procedures shows that real-time sequences can be obtained with either delivery method [19]. A variety of catheter models have been used in conjunction with the miniprobe (see Table 2). Access through the SpyGlass system (Boston Scientific, Natick, Massachusetts, USA), Olympus cholangioscopes (Olympus Corp., Tokyo, Japan), and Storz prototype cholangioscope (Karl Storz GmbH, Tuttlingen, Germany) are all also possible.

The Cellvizio CholangioFlex probe is dedicated to pCLE during ERCP procedures, and it has been miniaturized to an external diameter of $0.94 \mathrm{~mm}$, in order to meet the constraints of intraductal access devices. The probe fits inside lumens of $1.2 \mathrm{~mm}$ in diameter, has a field of view of $325 \mu \mathrm{m}$, a lateral resolution of 3.5 $\mu \mathrm{m}$, and an imaging depth of $40-70 \mu \mathrm{m}$ below the tissue surface. Specific pCLE image interpretation criteria are under development. Fig. 3 shows typical pCLE findings in normal bile ducts and biliary cancer.

\section{Colorectal disease \\ $\nabla$}

Several advanced imaging techniques may play a role in facilitating both the detectionof dysplasia in the colon, as well as classificationbetween neoplastic and non-neoplastic lesions during colonoscopy. CLE can provide imaging of single cells, making it unsuitable for the detection of lesions in the colon; however, it has 

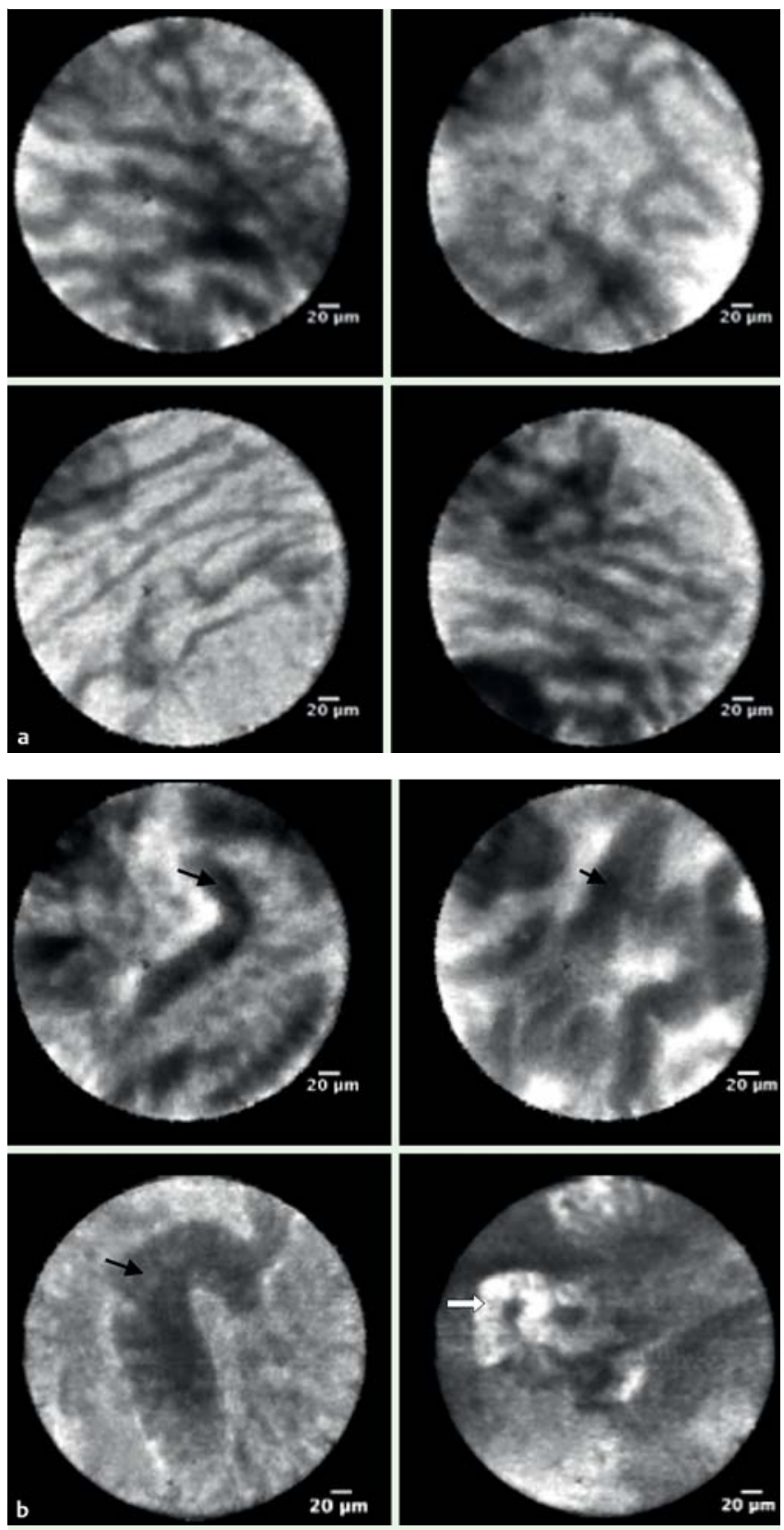

Fig. 3 Bile duct. a Normal appearing bile duct with fine, reticular gray pattern (thin black arrow). b Biliary cancer. Note dark, irregular structures (thin black arrow) interspersed with bright areas of tortuous dilated blood vessel (thick white arrow).

the potential to differentiate dysplasia from non-neoplastic tissue in a targeted approach $[2,20,21]$.

Chromoendoscopy, NBI, and a combination of NBI and AFI called endoscopic trimodal imaging (ETMI) may predict histology based on the Kudo pit pattern, vascular pattern intensity (VPI), and color. All of these techniques have a rather low specificity. CLE, however, has the potential to provide real-time conventional histopathology with a very high specificity.

Ideally, a method with a high detection rate of neoplastic lesions (usually at the cost of lower specificity) should be combined with a method of optimal differentiation of all detected lesions. Such combinations can be made by pan-colonic chromoendoscopy or autofluorescence as a "red flag technique" used in conjunction with endoscopic pit pattern analysis and CLE for the differentiation of detected lesions.

\section{Colorectal polyps}

$\nabla$

The detection of neoplastic lesions has been significantly improved by chromoendoscopy compared with conventional colonoscopy, whereas NBI only showed an improvement during the learning phase $[22,23]$. In one randomized study ETMI (white light $+\mathrm{NBI}+\mathrm{AFI}$ ) did not result in an increased adenoma detection rate [24].

Conventional endoscopy has limited ability to discriminate adenomatous from non-adenomatous colorectal polyps. In daily practice, all identified lesions are routinely removed and sent for histopathology. This approach results in substantial increase of endoscopic workload, additional pathology costs, and increase in potential complications. If CLE could reliably predict histology, then it could increase cost-effectiveness and efficiency considerably. This is a concept suggested by several prominent colonoscopists [25].

However, the decision to decide to leave a polyp in situ and not send it for pathology requires high accuracy and particularly high NPV of the imaging method. Chromoendoscopy, NBI, and AFI have shown unacceptable test characteristics for this purpose, with accuracies of $91 \%, 89 \%$, and $79 \%$, respectively [24]. CLE has shown high agreement with true histopathology. In earlier feasibility studies, Kiesslich et al. demonstrated an accuracy of $99 \%$ with the integrated system, whereas, feasibility studies with the probe-based system reached an accuracy of $82-92 \%$ $[2,20,21]$.

A prospective study of CLE in polyp differentiation has been published. Sanduleanu et al. performed a prospective study using chromoendoscopy-guided CLE with the integrated system to evaluate differential features of adenomatous and non-adenomatous colorectal polyps, resulting in an accuracy of 96\% [26]. Acriflavine was used in conjunction with fluorescein, enabling discrimination of HGD from LGD, with an accuracy of $97 \%$.

Another large prospective double-blind trial compared pCLE with NBI and Fujinon Intelligence Color Enhancement (FICE, Fujinon, Fort Wayne, New Jersey, USA), for polyp classification. Buchner et al. demonstrated that PCLE was superior to digital chromoendoscopy methods; however, it was primarily superior to FICE without significant improvement over NBI. The sensitivity of pCLE for neoplastic polyps was $91 \%$ and specificity was $76 \%$, compared with virtual chromoendoscopy (NBI and FICE), which had a sensitivity of $77 \%$ and a specificity of $71 \%$ [27].

This same group evaluated the learning curve for pCLE for colorectal polyps and found that new users rapidly acquired the ability to interpret pCLE images with accuracy similar to that of highly experienced experts. New users were able to interpret images with an accuracy of $93 \%$ after reading at least 35 cases. This study did not assess the accuracy of new users during live video endoscopies, which may require more experience [28].

Further prospective studies and data are eagerly awaited that examine the cost-effectiveness of CLE for colorectal polyps with both the integrated as well as the probe-based system. Data on the assessment of degree of dysplasia, inter-observer variability, and learning curve are also anticipated. At the same time, other applications for the use of the CLE system (e.g. early detection of adenomatous tissue at a scar at the resection site of a polyp), should be explored.

- Fig. 4 shows the criteria for pCLE classification of colorectal polyps. 


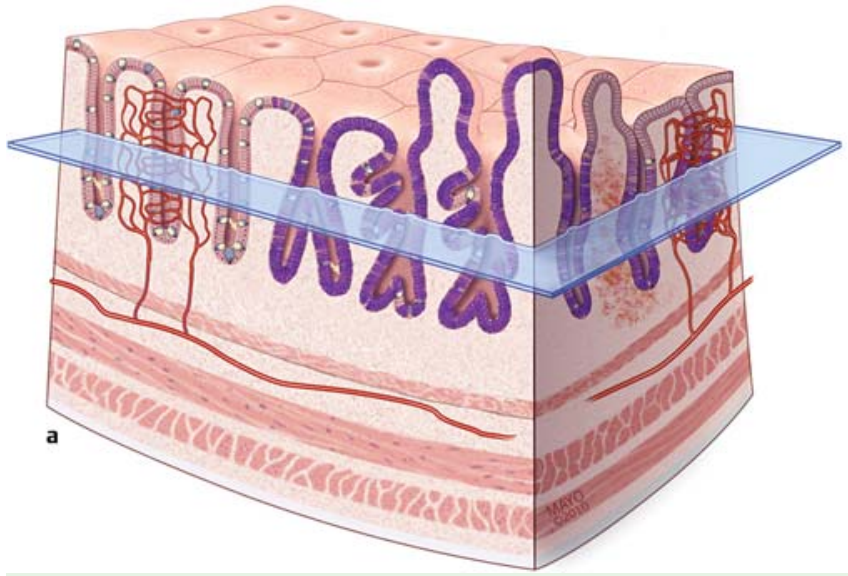

Fig. 4 Colon. a Graphic showing colonic mucosa progressing from normal crypts (left side) to dysplastic crypts and villiform structures (right side). b Panels showing probe-based confocal laser endomicroscopy images and hematoxylin and eosin images of colonic disease.

\section{Normal colon}

- Round crypt structures

- Dark goblet cells

(arrow)

- Regular, narrow vessels surrounding crypts (block arrow)
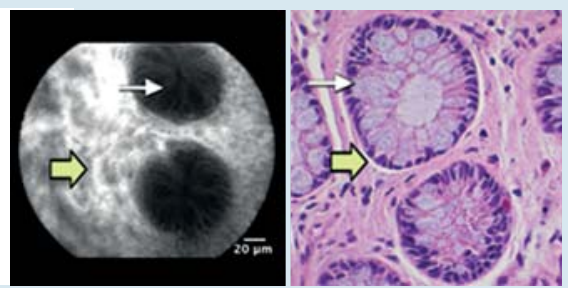

\section{Hyperplastic polyp}

- Crypts with slit or stellate openings (pits) - Bright non-thickened, uniform epithelium

- Dark „goblet“" cells (thin arrow)

- Small vessels (block arrow)
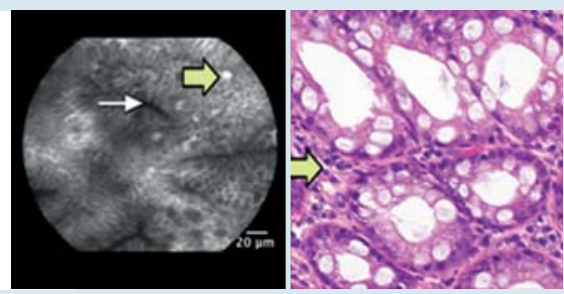

\section{Adenoma}

\section{- Irregular or villiform structures (note even \\ "tubular" adenoma \\ may have villiform structure on $\mathrm{pCLE}$ ) \\ - Dark, irregularly \\ thickened epithelium \\ - Decreased goblet cells}
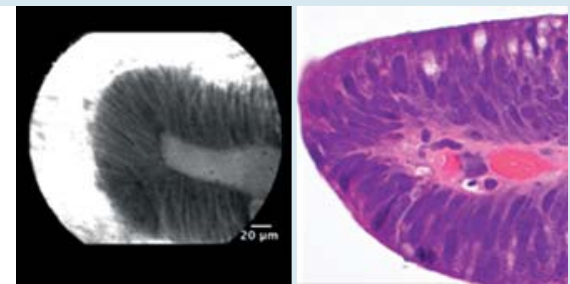

\section{Adenocarcinoma}

- Disorganized villiform or lack of structure

- Dark, irregularly

thickened epithelium

(thin arrow)

- Dilated vessels

(block arrow on H\&E)
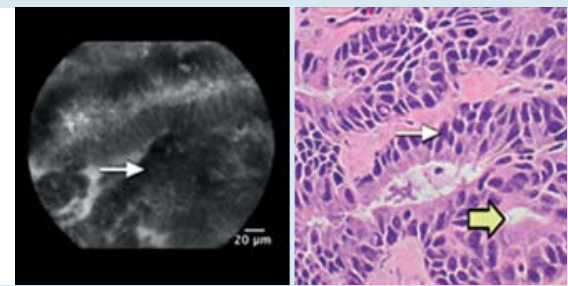

\section{Colitis}

\section{- Crypt fusion and distortion (arrow) - Bright epithelium - Dilated, prominent branching vessels (block arrow)}
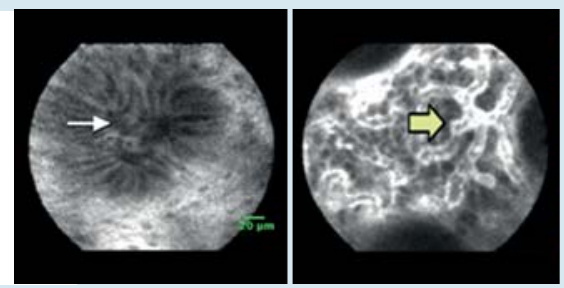


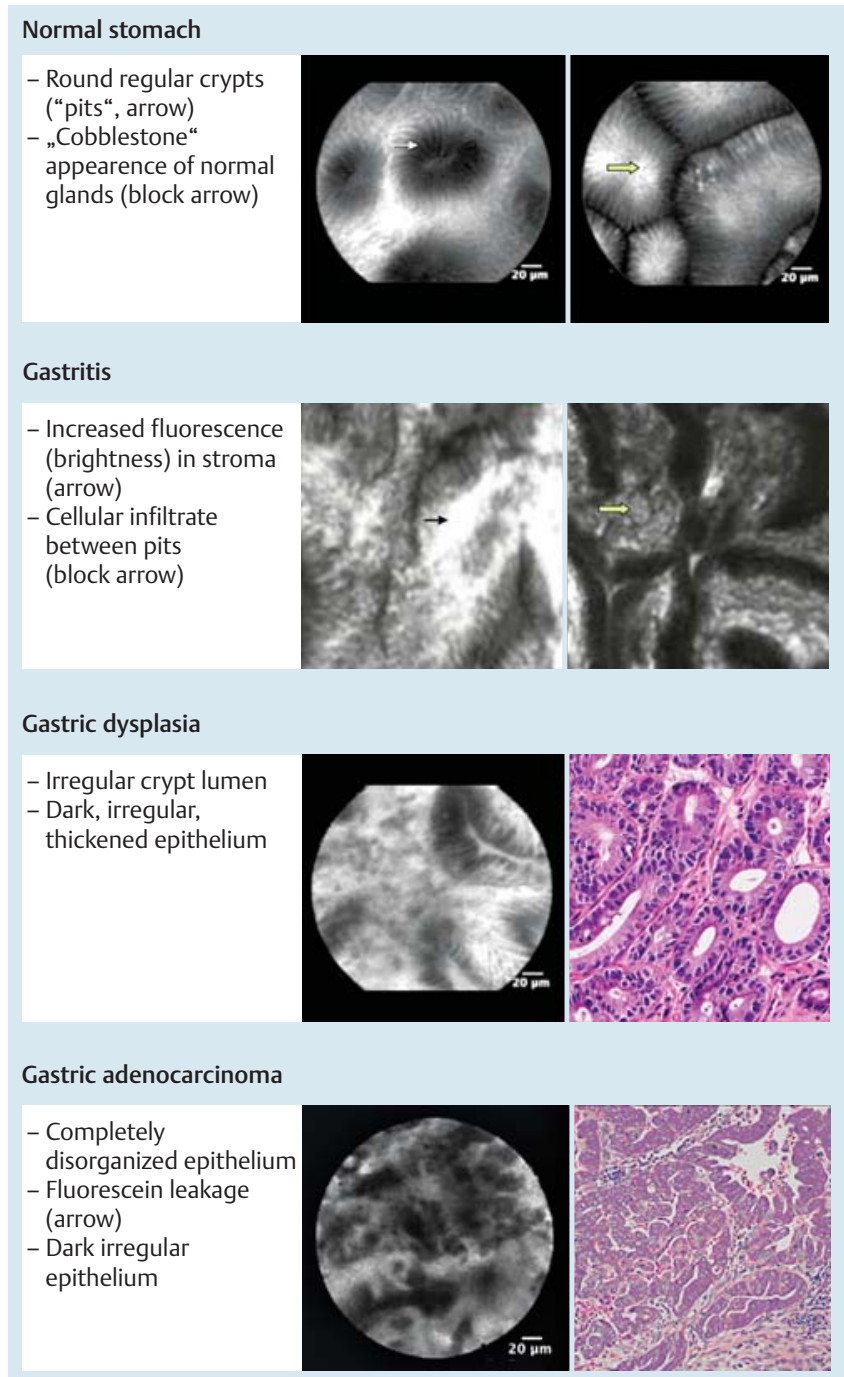

Fig. 5 Stomach. Panels showing probe-based confocal laser endomicroscopy images (normal, dysplasia, adenocarcinoma) and hematoxylin and eosin images of gastric disease. (Gastritis images kindly provided by Alexander Meining).

\section{Ulcerative colitis}

$\nabla$

As patients with ulcerative colitis have an increased risk of developing dysplasia, guidelines recommend colonoscopic surveillance including targeted biopsies of suspicious lesions and multiple (40) random biopsies. However, the yield for dysplasia on random biopsy is very low, and standard endoscopic imaging cannot identify most neoplastic lesions. Several randomized studies have shown that targeting biopsies with chromoendoscopy significantly increases dysplasia detection rates in patients with longstanding ulcerative colitis $[29,30]$. In one randomized study, ETMI also increased dysplasia detection rates but NBI did not $[31,32]$.

Using initial chromoscopy followed by directed eCLE combines the strengths of both techniques in ulcerative colitis. While panchromoscopy facilitates the detection of flat lesions in ulcerative colitis, subsequent targeted confocal endomicroscopy can be used to differentiate between neoplastic and non-neoplastic tissue, thus obviating the need for targeted biopsies of lesions that appeared non-neoplastic. This can also be used to possibly differentiate random biopsies in chromo-negative areas. By using this

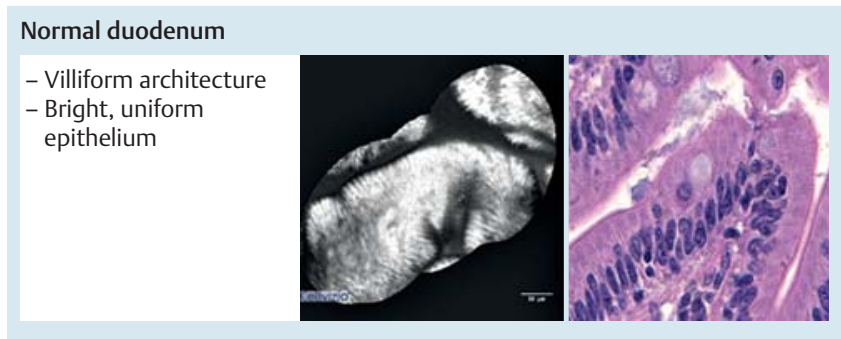

Duodenal adenoma

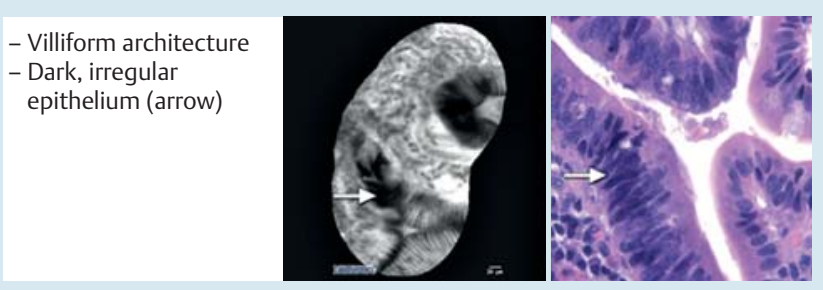

Duodenum celiac disease

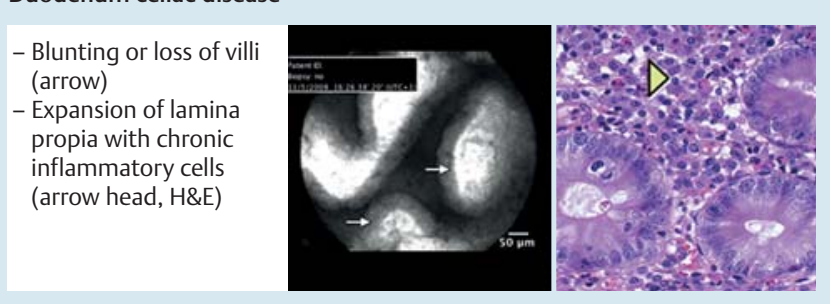

Fig. 6 Duodenum. Probe-based confocal laser endomicroscopy (pCLE) images and hematoxylin and eosin of normal and diseased duodenum. The top two panels show the high definition (UHD, Cellvizio, Mauna Kea Technologies, Paris) probe of normal and adenomatous duodenum. The field of view was expanded using "mosaicing" software, which integrates multiple side-by-side frames into a single panoramic view. The bottom pCLE image (celiac disease) was obtained using the Z probe (Cellvizio, Mauna Kea), which provides a lower-resolution but wider field of view.

diagnostic approach in a randomized study in 161 patients, Kiesslich et al. detected almost five times more dysplastic lesions than conventional colonoscopy with random biopsies [33].

Using the integrated CLE system, Hurlstone et al. demonstrated a high overall accuracy of $97 \%$ for in vivo differentiation of adenoma-like mass (ALM) and dysplasia-associated lesion or mass (DALM) in patients with chronic ulcerative colitis [34]. Case reports using the pCLE system suggest it may also be used to identify DALM lesions [35].This technique could provide gastroenterologists with important information for selecting patients who are suitable for immediate endoscopic resection vs. referral for pan-proctocolectomy. Recent studies also suggest that CLE may be a valuable tool for the grading of colitis and detection of microscopic colitis in regions of the colon without visible inflammation [36].

All of these studies have been performed with the integrated CLE system. Studies with the probe-based CLE system are currently underway. Further data from non-tertiary referral centers and from investigation of the learning curve and inter-observer agreement in patients with chronic ulcerative colitis are necessary before implementation of chromoscopy-guided CLE can be recommended in daily practice.

- Fig. 4 shows the criteria for pCLE classification of colitis and associated dysplasia. 


\section{Gastric diseases}

\section{$\nabla$}

To date there are limited data exploring application of confocal imaging to the stomach. There is a potential role for CLE in the discrimination of indeterminate gastric lesions in countries where gastric cancer is more common and screening endoscopies are performed. In two studies from Japan, CLE demonstrated typical features of dysplasia such as enlarged and increased numbers of nuclei [37]. CLE had an accuracy of 94-96\% for diagnosis of malignancy among 27 patients with early gastric cancer, when compared directly with histological biopsies [38]. Other potential but unexplored applications include distinction between adenomatous and fundic gland polyps. Fig. 5 shows typical pCLE images of normal gastric mucosa, inflammation, and dysplasia.

\section{Duodenal diseases}

As with the stomach, there are limited data on duodenal applications. Given the frequency of biopsies performed to detect celiac disease in patients with signs or symptoms of malabsorption, this would be a valuable role for CLE, especially given the relatively low yield of endoscopic biopsy. Two small case reports have suggested that confocal endomicroscopy can detect villous atrophy and increased intra-epithelial cellularity (presumed to be lymphocytes) $[39,40]$. In a recent study of 17 patients with celiac disease and 14 control individuals, CLE detected typical celiac changes of villous atrophy and crypt hypertrophy. The accuracy of CLE was excellent, with sensitivity of $94 \%$ and specificity of $92 \%[41]$.

Detection of duodenal adenomas is also a potentially valuable role for CLE. The risk of ampullary and non-ampullary duodenal cancer is substantially elevated in familial adenomatous polyposis, leading to recommendations for routine duodenal surveillance and ampullary biopsy [42]. A downside of biopsy of the ampulla is the risk of inducing acute pancreatitis [43], such that nonbiopsy methods would be preferable. Preliminary data from Shahid et al. at the Mayo Clinic (Florida, USA) suggest that pCLE may be highly accurate for the detection of both ampullary and nonampullary adenomas, although more data are needed [44]. The features of dysplasia are typical of those in other gastrointestinal sites including dark, irregular epithelium ( Fig. 6).

\section{Research priorities}

$\nabla$

To determine the value of these potential roles and explore new ones, critical research is needed, including in the following areas.

- Determine precise estimates of accuracy compared with current standard endoscopic methods in colon polyps, Barrett's esophagus, ulcerative colitis, biliary cancer, and gastric dysplasia.

- Determine the reliability of pCLE in a community setting.

- Assess the ability to guide immediate endoscopic therapy in Barretts-associated dysplasia, colorectal endoscopic mucosal resection, and polypectomy.

- Determine preliminary accuracy and reliability in bile duct cancer.

- Assess the cost-effectiveness of pCLE compared with standard competing technologies including biopsy.

- Develop improved methods for image stabilization.
- Develop improved methods for image interpretation systems including computer-aided diagnosis.

- Assess the feasibility and accuracy of intratumoral and intracystic CLE via needles (nCLE).

\section{Summary and future directions \\ $\nabla$}

pCLE has the potential to directly guide endoscopic therapy of dysplasia and significantly reduce the number of non-targeted (random) biopsies. Application of this technology has the further advantage of visualizing a dynamic process on a microscopic level for monitoring and determination of blood flow in various conditions. Further miniaturization of the probe will imply new indications, such as placing the miniprobe through an FNA needle for EUS-guided examinations of solid or cystic tumors and for interventions. In addition, integrating new biological fluorophores such as fluorescein-bound peptides will potentially enable the precise detection of dysplasia in vivo and guide subsequent intervention during the same procedure.

Competing interests: Dr Meining receives research funding from Mauna Kea Technologies and is co-patent holder of CholangioFlex probes. Dr Sharma receives funding from Barrx Inc., Cook Medical, Olympus, and Takeda. Dr Wallace receives research funding from Olympus, Cook Medical, Boston Scientific, Mauna Kea Technologies, and American BioOptics. Dr Dekker receives research funding from Olympus and has equipment on loan from Olympus and Mauna Kea Technologies. The other authors have nothing to declare.

\section{Institutions \\ ${ }^{1}$ Department of Gastroenterology, Mayo Clinic, Jacksonville, Florida, USA 2 Division of Surgical Pathology and Gastrointestinal Pathology Service, De- partment of Pathology, Massachusetts General Hospital and Harvard Medical School, Boston, Massachusetts, USA \\ ${ }^{3}$ Division of Gastroenterology and Hepatology, University of Colorado Denver, Denver, Colorado, USA \\ ${ }^{4}$ Academic Medical Center, Amsterdam, The Netherlands \\ ${ }^{5}$ Kansas University Medical Center and Department of Veterans Affairs Medical Center, Kansas City, Kansas, USA \\ ${ }^{6}$ Klinikum rechts der Isar, Technical University of Munich, Munich, Germany}

\section{Acknowledgment}

We thank Ms Alice McKinney of the Mayo Clinic Jacksonville Graphic Department for creation of the graphic representations of tissue histology, Ms Kelly Viola of the Mayo Clinic for editorial support, and Ms Anne Osdoit of Mauna Kea Technologies for providing confocal images and confirming technical aspects of the pCLE system.

\section{References}

1 Wang TD, Friedland S, Sahbaie P et al. Functional imaging of colonic mucosa with a fibered confocal microscope for real-time in vivo pathology. Clin Gastroenterol Hepatol 2007; 5: 1300-1305

2 Meining A, Bajbouj M, von Delius S, Prinz C. Confocal laser scanning microscopy for in vivo histopathology of the gastrointestinal tract. Arab J Gastroenterol 2007; 8: 14

3 Becker V, von Delius S, Bajbouj M et al. Intravenous application of fluorescein for confocal laser scanning microscopy: evaluation of contrast dynamics and image quality with increasing injection-to-imaging time. Gastrointest Endosc 2008; 68: 319-323

4 Kwan AS, Barry C, McAllister IL, Constable I. Fluorescein angiography and adverse drug reactions revisited: the Lions Eye experience. Clin Experiment Ophthalmol 2006; 34: 33-38 
5 Meining A, Wallace MB. Endoscopic imaging of angiogenesis in vivo. Gastroenterology 2008; 134: 915-918

6 Matysiak-Budnik T, Coron E, Mosnier J-F et al. 780 Quantitative in vivo assessment of vascular permeability in human colonic mucosa using confocal endomicroscopy: clinical implications for colonic neoplasia. Gastroenterology 2009; 136: A-123

7 Wang KK, Sampliner RE. Updated guidelines 2008 for the diagnosis, surveillance and therapy of Barrett's esophagus. Am J Gastroenterol 2008; 103: 788 - 797

8 Cameron AJ, Carpenter HA. Barrett's esophagus, high-grade dysplasia, and early adenocarcinoma: a pathological study. Am J Gastroenterol 1997; 92: 586-591

9 Kiesslich R, Gossner L, Goetz M et al. In vivo histology of Barrett's esophagus and associated neoplasia by confocal laser endomicroscopy. Clin Gastroenterol Hepatol 2006; 4: 979-987

10 Pohl H, Rosch T, Vieth M et al. Miniprobe confocal laser microscopy for the detection of invisible neoplasia in patients with Barrett's oesophagus. Gut 2008; 57: $1648-1653$

11 Uhlmann D, Wiedmann $M$, Schmidt $F$ et al. Management and outcome in patients with Klatskin-mimicking lesions of the biliary tree. J Gastrointest Surg 2006; 10: $1144-1150$

12 Wallace M, Abrams J, Bajbouj $M$ et al. Accuracy and inter-observer agreement of experts for probe-based confocal laser endomicroscopy detection of dysplasia in Barrett's esophagus. Gastrointest Endosc 2009; 69: AB351

13 Sharma P, Meining A, Coron E et al. Detection of neoplastic tissue in Barrett's esophagus with in vivo probe-based confocal endomicroscopy (DONT BIOPCE). Final results of a prospective international RCT: image guided versus 4 quadrant random biopsies?. Gastroenterology 2010; 138: S-155

14 Puhalla H, Schuell B, Pokorny $\mathrm{H}$ et al. Treatment and outcome of intrahepatic cholangiocellular carcinoma. Am J Surg 2005; 189: 173 - 177

15 Rosch T, Hofrichter K, Frimberger $E$ et al. ERCP or EUS for tissue diagnosis of biliary strictures? A prospective comparative study. Gastrointest Endosc 2004; 60: 390 - 396

16 Ponchon T, Gagnon P, Berger F et al. Value of endobiliary brush cytology and biopsies for the diagnosis of malignant bile duct stenosis: results of a prospective study. Gastrointest Endosc 1995; 42: 565-572

17 Schoefl R, Haefner M, Wrba F et al. Forceps biopsy and brush cytology during endoscopic retrograde cholangiopancreatography for the diagnosis of biliary stenoses. Scand J Gastroenterol 1997; 32: 363-368

18 Meining A, Saur D, Bajbouj M et al. In vivo histopathology for detection of gastrointestinal neoplasia with a portable, confocal miniprobe: an examiner blinded analysis. Clin Gastroenterol Hepatol 2007; 5: $1261-1267$

19 Stevens $P$, Chen $Y$, Shah R et al. Real-time intraductal confocal microscopy during ERCP: feasibility and technical considerations. Gastrointest Endosc 2009; 69: AB267

20 Kiesslich R, Burg J, Vieth $M$ et al. Confocal laser endoscopy for diagnosing intraepithelial neoplasias and colorectal cancer in vivo. Gastroenterology 2004; 127: 706-713

21 Becker V, Vercauteren T, von Weyhern $\mathrm{CH}$ et al. High-resolution miniprobe-based confocal microscopy in combination with video mosaicing (with video). Gastrointest Endosc 2007; 66: 1001 - 1007

22 Adler A, Pohl H, Papanikolaou IS et al. A prospective randomised study on narrow-band imaging versus conventional colonoscopy for adenoma detection: does narrow-band imaging induce a learning effect? Gut 2008; 57: 59-64

23 Adler A, Aschenbeck J, Yenerim T et al. Narrow-band versus white-light high definition television endoscopic imaging for screening colonoscopy: a prospective randomized trial. Gastroenterology 2009; 136: $410-$ 416

24 van den Broek FJ, van Soest EJ, Naber AH et al. Combining autofluorescence imaging and narrow-band imaging for the differentiation of adenomas from non-neoplastic colonic polyps among experienced and non-experienced endoscopists. Am J Gastroenterol 2009; 104: $1498-1507$

25 Ignjatovic A, East JE, Suzuki N et al. Optical diagnosis of small colorectal polyps at routine colonoscopy (Detect InSpect ChAracterise Resect and
Discard; DISCARD trial): a prospective cohort study. Lancet Oncol 2009; 10: $1171-1178$

26 Sanduleanu S, Driessen A, Gomez-Garcia E et al. In vivo diagnosis and classification of colorectal neoplasia by chromoendoscopy-guided confocal laser endomicroscopy. Clin Gastroenterol Hepatol 2010; 8: $371-378$

27 Buchner AM, Shahid MW, Heckman MG et al. Comparison of probebased confocal laser endomicroscopy with virtual chromoendoscopy for classification of colon polyps. Gastroenterology 2010; 138: 834842

28 Buchner AM, Gomez V, Heckman MG et al. The learning curve of in vivo probe-based confocal laser endomicroscopy for prediction of colorectal neoplasia. Gastrointest Endosc 2011; 73: 556-560

29 Kiesslich R, Fritsch J, Holtmann M et al. Methylene blue-aided chromoendoscopy for the detection of intraepithelial neoplasia and colon cancer in ulcerative colitis. Gastroenterology 2003; 124: 880-888

30 Rutter MD, Saunders BP, Schofield G et al. Pancolonic indigo carmine dye spraying for the detection of dysplasia in ulcerative colitis. Gut 2004; 53: 256-260

31 van den Broek FJ, Fockens P, van Eeden $S$ et al. Endoscopic tri-modal imaging for surveillance in ulcerative colitis: randomised comparison of high-resolution endoscopy and autofluorescence imaging for neoplasia detection; and evaluation of narrow-band imaging for classification of lesions. Gut 2008; 57: 1083-1089

32 Dekker E, vandenBroek FJ, Reitsma JB et al. Narrow-band imaging compared with conventional colonoscopy for the detection of dysplasia in patients with longstanding ulcerative colitis. Endoscopy 2007; 39: $216-221$

33 Kiesslich R, Goetz M, Lammersdorf $K$ et al. Chromoscopy-guided endomicroscopy increases the diagnostic yield of intraepithelial neoplasia in ulcerative colitis. Gastroenterology 2007; 132: 874-882

34 Hurlstone DP, Thomson M, Brown S et al. Confocal endomicroscopy in ulcerative colitis: differentiating dysplasia-associated lesional mass and adenoma-like mass. Clin Gastroenterol Hepatol 2007; 5: 1235 1241

35 Palma GD, Staibano S, Siciliano S et al. In-vivo characterization of DALM in ulcerative colitis with high-resolution probe-based confocal laser endomicroscopy. World J Gastroenterol 2011; 17: 677-680

$36 \mathrm{Li} \mathrm{CQ}$ Xie XJ, Yu Tet al. Classification of inflammation activity in ulcerative colitis by confocal laser endomicroscopy. Am J gastroenterol 2010; 105: $1391-1396$

37 Kakeji Y, Yamaguchi S, Yoshida D et al. Development and assessment of morphologic criteria for diagnosing gastric cancer using confocal endomicroscopy: an ex vivo and in vivo study. Endoscopy 2006; 38 : $886-890$

38 Kitabatake S, Niwa Y, Miyahara R et al. Confocal endomicroscopy for the diagnosis of gastric cancer in vivo. Endoscopy 2006; 38: 1110 1114

39 Trovato C, Sonzogni A, Ravizza D et al. Celiac disease: in vivo diagnosis by confocal endomicroscopy. Gastrointest Endosc 2007; 65: 10961099

40 Zambelli A, Villanacci V, Buscarini E et al. Confocal laser endomicroscopy in celiac disease: description of findings in two cases. Endoscopy 2007; 39: $1018-1020$

41 Leong $R W$, Nguyen NQ, Meredith CG et al. In vivo confocal endomicroscopy in the diagnosis and evaluation of celiac disease. Gastroenterology 2008; 135 : $1870-1876$

42 Vasen HF, Moslein G, Alonso A et al. Guidelines for the clinical management of familial adenomatous polyposis (FAP). Gut 2008; 57: 704 - 713

43 Nugent KP, Spigelman $A D$, Williams $C B$, Phillips $R K$. latrogenic pancreatitis in familial adenomatous polyposis. Gut 1993; 34: 1269-1270

44 Shahid M, Buchner A, Hasan $M$ et al. The role of probe-based confocal laser endomicroscopy (pCLE) in detection of dysplasia in duodenal polyps. Gastrointest Endosc 2009; 69: AB369 